\title{
Assessment of Anemia and Lipid Metabolism Disorder Among Baryte Mine Employees
}

\author{
Umesh Dhumne, Sarang V Dhatrak*, Subroto S Nandi and Shilpa Ingole \\ Department of Occupational Health, National Institute of Miners' Health, India \\ *Corresponding author: Sarang V Dhatrak, Department of Occupational Health, National Institute of Miners' Health, India
}

\section{ARTICLE INFO}

Received: 㗀 February 13, 2020

Published: 慧 February 27, 2020

Citation: Umesh Dhumne, Sarang V Dhatrak, Subroto S Nandi, Shilpa Ingole. Assessment of Anemia and Lipid Metabolism Disorder Among Baryte Mine Employees. Biomed J Sci \& Tech Res 26(1)2020. BJSTR. MS.ID.004294.

Keywords: Anemia; ATP III; Dyslipidemia; Mine Employees

Abbreviations: ATP: Adult Treatment Panel; HDL: High-Density Lipoprotein; LDL: Low-Density Lipoprotein; WHO: World Health Organization; T2DM: Type 2 Diabetes Mellitus; CVD: Cardiovascular Disease; CHD: Coronary Heart Disease; BMI: Body Mass Index; TC: Total Cholesterol; HDL-C: High Density Lipoprotein Cholesterol; LDL-C: Low Density Lipoprotein Cholesterol; TG: Triglyceride

\section{ABSTRACT}

Background: Mangampet baryte mine is a famous for world's largest source of barytes. Mineral is richly available in YSR Kadapa district and is widely used in several industries e.g. oil, glass, paint and chemical industries. Work related stress is linked to the risk of suffering cardiovascular disease as a result of unhealthy habits such as smoking, drinking and unsuitable diet. The anemia is usually mild in degree and presents serious clinical problems. Hence hemoglobin and lipid concentration were evaluated.

Objective: The present study was designed to assess the prevalence of anemia and dyslipidemia among baryte mine employees of Andhra Pradesh, India.

Materials and methods: This study was performed among the baryte mine employees in 18-60 years of age group. A total of 415 employees were participated in the present study. Blood samples were collected for the estimation of anemia and lipid moieties. Anemia and dyslipidemia were classified as per the World Health Organization (WHO) and Adult Treatment Panel (ATPIII) grading criteria respectively.

Results: The overall prevalence of anemia among these mine employees was $48.4 \%$. The majority $166(82.5 \%)$ showed mild anemia while moderate anemia was recorded in $32(15.9 \%)$ and $3(1.4 \%)$ mine employees had severe anemia. Hypercholesterolemia, hypertriglyceridemia and low High-Density Lipoprotein (HDL) was present in 47.7\%, $63.3 \%$ and $6.0 \%$ in the baryte mine employees respectively. Among the age group the serum levels of lipid indices were higher in 18 - 30 years of age group. Dyslipidemia shows the highest prevalence of hypertriglyceridemia was seen in present study. The prevalence of Low-Density Lipoprotein (LDL) cholesterol was recorded in $33.0 \%$ of the mine employees.

Conclusion: The study highlighted the high prevalence of anemia and dyslipidemia among these mine employees and there is urgent need for managing anemia and hypertriglyceridemia. Study findings suggest that there is a need to arise educating the mine employees behavior on diet, physical activities and further surveillance in these mine employees is essential for verifying the impact of anemia and dyslipidemia.

\section{Introduction}

Workers are exposed to a wide variety of hazardous substances at their workplace and suffer from occupational and nonoccupational serious medical illness. Anemia is one of the most common health problems in India which is much more prevalent in the rural than in the urban areas [1,2]. Globally, anemia affects 1.62 billion people, which corresponds to $24.8 \%$ of the population [3]. In 1992, World Health Organization (WHO) global estimates of anemia prevalence averaged $56 \%$, with a range of $35-75 \%$ depending on geographic location (WHO, 1992). Prevalence of anemia in South Asia is among the highest in the world, mirroring overall high rates of malnutrition.

Dyslipidemia signifies the increased concentration of total cholesterol and LDL cholesterol, decreased concentration of HDL cholesterol and hypertriglyceridemia present alone or in combination. A combination of lipid abnormalities, hypertriglyceridemia and low HDL, are metabolically interlinked 
and have been termed as "atherogenic dyslipidemia" [4,5]. This pattern of dyslipidemia has shown a strong association with Type 2 Diabetes Mellitus (T2DM) and Cardiovascular Disease (CVD) in several studies in developed countries [5,6]. Psychosocial stress increases serum cholesterol causes hypertension and enhances clot formation. Cigarette smoking is another risk factor for Coronary Heart Disease (CHD). Other occupational factors related to CHD are sedentary work, exposure to carbon disulfide, carbon monoxide and nitrates and chromic exposure to noise, heat and cold. Approximately $25.0 \%$ of all deaths in developed countries are due to CHD (Baxter and Petch, 1995: 270). There are many risk factors for CHD of non-occupational origin, which include hypertension (high blood pressure), smoking, diet, hypercholesterolemia (raised cholesterol) and obesity. These risk factors can work in a synergistic way with occupational exposure, which increases the risk of developing CHD. Many studies in southern India have reported high prevalence of anemia and dyslipidemia. But anemia study mainly carried out among women and study on dyslipidemia was carried out in rural and urban population. Reliable data on the prevalence of anemia and dyslipidemia among mine employees is not much available, particularly among the baryte mine employees of Andhra Pradesh, India. Thus, the present study was undertaken to investigate the prevalence of anemia and dyslipidemia among the baryte mine employees.

\section{Material and Methods}

Present study was conducted among baryte mine employees during August 2012 to October 2012. A random sampling method was adopted. All of 415 mine employees, working in different occupations were considered for the study. The data was collected from all the study subjects and analyzed. The personal data on age, sex, work exposure and occupations were noted. Body weight and height were measured according to standardized technique and calibrated equipment's. Body Mass Index (BMI) was calculated as weight in kilograms divided by squared height in meter $\left(\mathrm{kg} / \mathrm{m}^{2}\right)$. Fasting blood samples was collected from the brachial vein of which $2.5 \mathrm{ml}$ was dispensed in a $5 \mathrm{ml}$ sterile test tube containing EDTA (Ethylene Diamine Tetra acetic acid) as an anticoagulant, for the analysis of hemoglobin concentration. The remaining $2.5 \mathrm{ml}$ blood was dispensed in a plain sterile test tube and allowed for 10 minutes to clot at room temperature. Hemoglobin estimation was done by the cyanmethemoglobin method. The lipid profile included the following indices: Total Cholesterol (TC), High Density Lipoprotein Cholesterol (HDL-C), Low Density Lipoprotein Cholesterol (LDL-C), Triglyceride (TG) and TC/HDL-C ratio. The assessment of the lipid profile was analyzed by using standard laboratory techniques on evolution 3000 (TULIP) semi auto analyzer. LDL- cholesterol was determined from the values of total cholesterol and HDL cholesterol using Fried Wald's formula.

The classification of anemia was based upon criteria developed by the WHO. Mild anemia was defined as hemoglobin concentration between 11 to $12.9 \mathrm{~g} / \mathrm{dl}$ for male and 11 to $11.9 \mathrm{~g} / \mathrm{dl}$ for female, moderate anemia was defined as 8 to $10.9 \mathrm{~g} / \mathrm{dl}$ for male and female, severe anemia was defined as less than $8 \mathrm{~g} / \mathrm{dl}$ for male and female. Adult Treatment Panel III (ATP III) was used for the classification of the serum levels of each lipid parameters. Data entry and analysis were done by using Epi info statistical software. Proportion, standard deviation and chi square test were used for analysis.

\section{Results}

Table 1 shows demographic characteristics of study subjects according to gender, age, BMI and smoking history. It is seen that majority 357 (86 \%) of study subjects were males and only 58 (13.9\%) were females. Out of 415 mine employees, 267 (64.3\%) were in the age group of 18-40 years while only 148 (35.6\%) belonged to age group of 41-60 years. 27 (6.5\%) mine employees were having low $(<18.5)$ level of BMI, about 178 (42.8\%) were normal (18.5-24.9) level of BMI and 210 (50.6\%) mine employees were having high $(\geq 25)$ level of BMI. Most of the mine employees were nonsmokers (82.8\%) while $17.1 \%$ employees were noted smoking history.

Table 1: Demographic characteristics of the study subjects.

\begin{tabular}{|c|c|}
\hline Variables & No. of subjects (\%) \\
\hline Gender & $357(86.0)$ \\
\hline Male & $58(13.9)$ \\
\hline Female & \\
\hline Age Group (yrs) & $151(36.3)$ \\
\hline $18-30$ & $116(27.9)$ \\
\hline $31-40$ & $71(17.1)$ \\
\hline $41-50$ & $77(18.5)$ \\
\hline $51-60$ & $27(6.5)$ \\
\hline BMI & $178(42.8)$ \\
\hline Underweight & $165(39.7)$ \\
\hline Normal & $45(10.8)$ \\
\hline Overweight & \\
\hline Obese & $71(17.1)$ \\
\hline Smoking Habits & $344(82.8)$ \\
\hline Smokers & \\
\hline Non-Smokers & \\
\hline & \\
\hline & \\
\hline
\end{tabular}

Sexwise severity of anemia among mine employees is shown in Table 2. The overall prevalence of anemia among baryte mine employees was 48.4\%. The majority 166 (82.5\%) demonstrated mild anemia while moderate and severe anemia was recorded in $15.9 \%$ \& $1.4 \%$ respectively $(\mathrm{p}<0.001)$. The findings show that moderate anemia (59.3\%) was higher among the male employees than that of mild anemia (57.6\%) and severe anemia (1.7\%). Similarly, moderate anemia (40.6\%) was higher among the female employees than that of mild anemia (9.6\%). None of the female employees had severe anemia. The association of anemia with age group is shown in Table 3. In different age groups, out of 201 anemic mine employees, majority (61.6\%) of the mine employees were anemic in the age group of $18-40$ years while $38.3 \%$ mine 
employees having anemia in the age group 41-60 years. There was no statistically significant difference among the age groups.

Table 2: Sexwise severity of anemia among mine employees.

\begin{tabular}{|c|c|c|c|}
\hline \multirow{2}{*}{ Anemia Classification } & \multicolumn{2}{|c|}{ No. of Subjects } & \multirow{2}{*}{ Total } \\
\cline { 2 - 3 } & Male & Female & \\
\hline Mild & $150(57.6)$ & $16(9.6)$ & $166(82.5)$ \\
\hline Moderate & $19(59.3)$ & $13(40.6)$ & $32(15.9)$ \\
\hline Severe & $3(1.7)$ & 0 & $3(1.4)$ \\
\hline Total & $172(85.5)$ & $29(14.4)$ & $201(48.4)$ \\
\hline
\end{tabular}

Table 3: Distribution of anemia according to age group.

\begin{tabular}{|c|c|c|c|c|c|c|c|}
\hline \multirow{2}{*}{ Age Group (yrs) } & \multicolumn{2}{|c|}{ Anemic } & \multicolumn{2}{c|}{ Non-Anemic } & \multicolumn{2}{c|}{ Total } & \multirow{2}{*}{ p } \\
\cline { 2 - 7 } & $\mathbf{n}$ & $\mathbf{\%}$ & $\mathbf{n}$ & $\mathbf{\%}$ & $\mathbf{n}$ & $\mathbf{\%}$ & \\
\hline $18-30$ & 67 & 44 & 85 & 55.9 & 152 & 36.6 & \\
\cline { 1 - 7 } $31-40$ & 57 & 48.7 & 60 & 51.2 & 117 & 28.1 & \multirow{2}{*}{0.078} \\
\cline { 1 - 6 } $41-50$ & 31 & 43.6 & 40 & 56.3 & 71 & 17.1 & \\
\hline $51-60$ & 46 & 61.3 & 29 & 38.6 & 75 & 18 & \\
\hline Total & 201 & 48.4 & 214 & 51.5 & 415 & 100 & \\
\hline
\end{tabular}

Distribution of mine employees with total cholesterol level as per ATP III is shown in Table 4. Most of the male (48.1\%) and female (77.5\%) employees were in desirable group. Out of 415 employees 119 (28.6\%) showed total cholesterol levels between 200 to 239 $\mathrm{mg} / \mathrm{dl}$. The remaining 19\% employees were in the risk group having total cholesterol more than or equal to $240 \mathrm{mg} / \mathrm{dl}$. There was a significant difference $(\mathrm{p}<0.0001)$ in total cholesterol level between male and female employees. As per ATP III triglyceride levels among the employees according to sex are shown in Table 5. Only 31.6\% of male employees were having normal triglyceride level while most $(67.2 \%)$ of the female employees were having normal triglyceride level. The remaining majority (63.3\%) of the mine employees were in the risk group having hypertriglyceridemia, of which $68.3 \%$ and $32.7 \%$ workers were male and female respectively. There was a significant difference $(\mathrm{p}<0.0001)$ in triglyceride level between male and female employees. None of the mine employees had very high level of triglyceride.

Table 4: Sexwise severity of anemia among mine employees.

\begin{tabular}{|c|c|c|c|}
\hline \multirow{2}{*}{$\begin{array}{c}\text { Total Cholesterol Level } \\
(\mathbf{m g} / \mathbf{d l})\end{array}$} & \multicolumn{2}{|c|}{ No. of Employees } & \multirow{2}{*}{ Total } \\
\cline { 2 - 3 } & Male & Female & \\
\hline Desirable $(<200)$ & $172(48.1)$ & $45(77.5)$ & $217(52.2)$ \\
\hline Moderate High $(200-239)$ & $112(31.3)$ & $7(12.0)$ & $119(28.6)$ \\
\hline High ( $\geq 240)$ & $73(20.4)$ & $6(10.3)$ & $79(19.0)$ \\
\hline Total & $357(100)$ & $58(100)$ & $415(100)$ \\
\hline
\end{tabular}

Table 5: Sexwise distribution of employees with triglyceride level as per ATPIIII.

\begin{tabular}{|c|c|c|c|}
\hline \multirow{2}{*}{ Triglyceride Level (mg/dl) } & \multicolumn{2}{|c|}{ No. of Employees } & \multirow{2}{*}{ Total } \\
\cline { 2 - 3 } & Male & Female & \\
\hline Normal $(<150)$ & $113(31.6)$ & $39(67.2)$ & $152(36.6)$ \\
\hline Moderate $(150-199)$ & $194(54.3)$ & $14(24.1)$ & $208(50.1)$ \\
\hline
\end{tabular}

\begin{tabular}{|c|c|c|c|}
\hline High $(200-499)$ & $50(14.0)$ & $5(8.6)$ & $55(13.2)$ \\
\hline Very High $(>500)$ & 0 & 0 & 0 \\
\hline Total & $357(100)$ & $58(100)$ & $415(100)$ \\
\hline
\end{tabular}

Table 6 shows HDL cholesterol levels among study subjects. Low level of HDL cholesterol was observed among 5.8\% in male and $6.8 \%$ in female employees. The remaining majority (93.9\%) of the mine employees were having moderate and high level of HDL cholesterol that means the desirable level, thus the risk group consisted only $6 \%$ of the mine employees. However, the difference between the male and female employees was not statistically significant. Age wise prevalence of dyslipidemia among mine employees is shown in Table 7. The studied mine employees showed a very high prevalence of dyslipidemia among 18 -30 years of age group as compare to other age groups. Hypertriglyceridemia showed the highest prevalence (63.3\%) while low level of HDL cholesterol level in $6.0 \%$ of the employees. The prevalence of total cholesterol and LDL cholesterol was recorded $47.7 \%$ and $33.0 \%$ respectively.

Table 6: HDL cholesterol levels among study subjects as per ATP III.

\begin{tabular}{|c|c|c|c|}
\hline \multirow{2}{*}{$\begin{array}{c}\text { HDL Cholesterol (mg/ } \\
\text { dl) }\end{array}$} & \multicolumn{2}{|c|}{ No. of Subjects } & \multirow{2}{*}{ Total } \\
\cline { 2 - 3 } & Male & Female & \\
\hline Low $(<40)$ & $21(5.8)$ & $4(6.8)$ & $25(6.0)$ \\
\hline Moderate $(41-60)$ & $302(84.5)$ & $48(82.7)$ & $350(84.3)$ \\
\hline High (> 60) & $34(9.5)$ & $6(10.3)$ & $40(9.6)$ \\
\hline Total & $357(100)$ & $58(100)$ & $415(100)$ \\
\hline
\end{tabular}

Table 7: Age wise prevalence of dyslipidemia among mine employees.

\begin{tabular}{|c|c|c|c|c|}
\hline \multirow{2}{*}{ Age Group (yrs) } & \multicolumn{4}{|c|}{ Prevalence of Dyslipidemia } \\
\cline { 2 - 5 } & TC & TG & HDL-C & LDL-C \\
\hline $18-30(n=152)$ & $77(50.6)$ & $99(65.1)$ & $6(3.9)$ & $50(32.8)$ \\
\hline $31-40(n=117)$ & $53(45.2)$ & $73(62.3)$ & $4(3.4)$ & $36(30.7)$ \\
\hline $41-50(n=71)$ & $31(43.6)$ & $43(60.5)$ & $7(9.8)$ & $27(38.0)$ \\
\hline $51-60(n=75)$ & $37(49.3)$ & $48(64.0)$ & $8(10.6)$ & $24(32.0)$ \\
\hline Total $(n=415)$ & $198(47.7)$ & $263(63.3)$ & $25(6.0)$ & $137(33.0)$ \\
\hline
\end{tabular}

\section{Discussion}

Anemia and dyslipidemia are a major public health problem throughout the world. Study on prevalence of anemia and dyslipidemia among the baryte mine workers of Andhra Pradesh is nonexistence. The present study was undertaken in order to estimate the prevalence of anemia and dyslipidemia among baryte mine workers of Andhra Pradesh, India. The present study demonstrated that the overall prevalence of anemia was $48.4 \%$. In present study $82.5 \%$ had mild anemia while $15.9 \%$ were moderately anemia and only $1.4 \%$ had severe anemia. According to WHO if the prevalence of anemia at community levels is more than $40 \%$ it is considered as problem of high magnitude [2]. In the present study, the prevalence of hypercholesterolemia, hypertriglyceridemia and elevated LDL cholesterol was observed 
to be higher in the 18-30 years of age group compared to the other age group. However, the prevalence low HDL cholesterol level was higher among 51-60 years of age group. The lower prevalence of dyslipidemia was observed among 41-50 years of age group. Serum cholesterol and Coronary Artery Disease (CAD) have been shown to have a consistent and strong relationship [7]. Additionally, a large number of clinical trials have provided indisputable evidence that lowering cholesterol levels decreases CAD events [8]. Mean serum cholesterol levels $>200 \mathrm{mg} / \mathrm{dl}$ have been shown to be a risk factor for CAD [9]. Present study found that the hypercholesterolemia $(47.7 \%)$ was the second highest prevalence of dyslipidemia.

In our study, $63.3 \%$ of the mine employees had elevated level of triglyceride. Several studies have reported a strong association of CAD with serum triglycerides [10]. There was also a significant difference in the prevalence of high risk of triglyceride levels between male and female groups. In India the overall prevalence of dyslipidemia in various studies ranges from $10 \%$ to $73 \%$ [11]. The results from a recent cross-sectional study in urban New Delhi (North India) showed the prevalence of hypertriglyceridemia to be $42.7 \%$ [12]. There is paucity of studies on anemia and dyslipidemia in baryte mine employees of Andhra Pradesh. However, it is difficult to compare observations of the various studies due to different sampling procedures and different population sample.

\section{Conclusion}

The present study found that a high prevalence of anemia, total cholesterol, triglycerides as well as low HDL cholesterol among baryte mine employees of Andhra Pradesh. Our study highlights the prevalence of anemia was more in all the age groups and the prevalence of dyslipidemia was more prevalent in younger age groups. Study confirmed that anemia and dyslipidemia is a major health problem in baryte mine employees of Andhra Pradesh. Hence, the study suggests that there is a need to arise educating the mine workers behavior on diet, physical activities and further surveillance in these mine employees is essential for verifying the impact of anemia and dyslipidemia.

\section{References}

1. (1998) National Consultation on the Control of Nutritional Anemia in India Department of Family Welfare (Maternal Health Division), Ministry of Health and Family Welfare, Nirman Bhawan, New Delhi, India.

2. Seshadri S (1999) A database on Iron Deficiency Anaemia (IDA) in India: prevalence, causes, Consequences and strategies for prevention. Department of Foods and Nutrition. WHO Collaborating Centre for Nutrition Research. The Maharaja Sayajirao University of Baroda, Vadodara, India.

3. de Benoist B, McLean E, Egli I, Cogswell M (2008) Worldwide prevalence of anaemia 1993-2005: WHO global database on anaemia.

4. Grundy SM, Vega GL (1992) Two different views of the relationship of hyper-triglyceridemia to coronary heart disease: Implications for treatment. Arch Intern Med 152(1): 28-34.

5. Vega GL (2004) Management of atherogenic dyslipidemia of the metabolic syndrome: Evolving rationale for combined drug therapy. Endocrinol. Metab Clin N Am 33(3): 525-544.

6. Chandalia M, Deedwania PC (2001) Coronary heart disease and risk factors in Asian Indians. Adv Exp Med Biol 498: 27-34.

7. Gotto AM (1997) Cholesterol management in theory and practice. Circulation 96(12): 4424-4430.

8. Waters DD (2001) What do the statin trials tell us? Am J Manag Care 7: S138-S143.

9. Mathur SK, Wahi PN, Malhotra KK, Sharma RD, Srivastava SK (1959) Dietary fats, serum cholesterol and lipids in different socioeconomic groups in Uttar Pradesh. J Indian Med Assoc 33: 303-309.

10. Austin MA, Hokanson JE, Edwards KL (1998) Hypertriglyceridemia as a cardiovascular risk factor. Am J Cardiol 81(4A): 7B-12B.

11. Misra A, Luthra K, Vikram NK (2004) Dyslipidemia in Asian Indians: Determinants and significance. J Assoc Physicians India 52: 137-142.

12. Bhardwaj S, Misra A, Misra R, Goel K, Bhatt SP, et al. (2011) High prevalence of abdominal, intra-abdominal and subcutaneous adiposity and clustering of risk factors among urban Asian Indians in North India. PLoS One 6(9): e24362.

\section{ISSN: 2574-1241}

DOI: 10.26717/BJSTR.2020.26.004294

Sarang V Dhatrak. Biomed J Sci \& Tech Res

This work is licensed under Creative Commons Attribution 4.0 License

Submission Link: https://biomedres.us/submit-manuscript.php

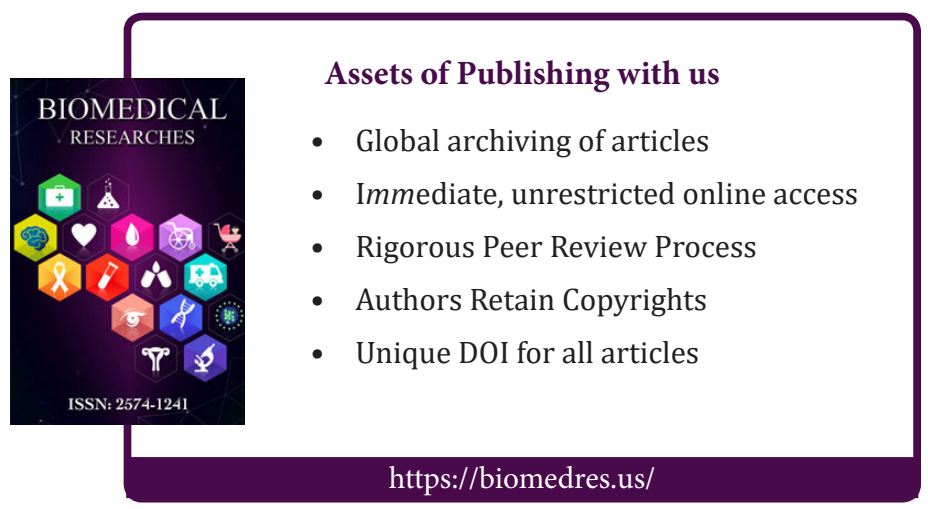

